\title{
Erratum to: Gliomatosis cerebri: no evidence for a separate brain tumor entity
}

\author{
Ulrich Herrlinger ${ }^{1}$ David T. W. Jones ${ }^{2,3} \cdot$ Martin Glas $^{1,4}$ • Elke Hattingen ${ }^{5}$ \\ Dorothee Gramatzki $^{6}$ - Moritz Stuplich ${ }^{1}$ Jörg Felsberg ${ }^{7}$ - Oliver Bähr ${ }^{15}$. \\ Gerrit H. Gielen ${ }^{8} \cdot$ Matthias Simon $^{9}$ Dorothee Wiewrodt ${ }^{16}$ • Martin Schabet ${ }^{17}$. \\ Volker Hovestadt ${ }^{3,10} \cdot$ David Capper ${ }^{3,11,12} \cdot$ Joachim P. Steinbach $^{15}$. \\ Andreas von Deimling, $3,11,12 \cdot$ Peter Lichter ${ }^{3,10} \cdot$ Stefan M. Pfister ${ }^{2,3,13}$. \\ Michael Weller $^{6} \cdot$ Guido Reifenberger $^{7,14}$
}

Published online: 7 January 2016

(C) Springer-Verlag Berlin Heidelberg 2016

\section{Erratum to: Acta Neuropathol \\ DOI 10.1007/s00401-015-1495-z}

The original version of this article contained errors in the alignment of several entries in Tables 4 and 5. The corrected Tables 4 and 5 are given below. The original article has been updated accordingly.

The online version of the original article can be found under doi:10.1007/s00401-015-1495-z.

Ulrich Herrlinger

ulrich.herrlinger@ukb.uni-bonn.de

1 Division of Neurooncology, Department of Neurology, University Medical Center Bonn, Sigmund-Freud-Str. 25, 53127 Bonn, Germany

2 Division of Pediatric Neurooncology, German Cancer Research Center (DKFZ) Heidelberg, Heidelberg, Germany

3 German Cancer Consortium (DKTK), Core Center Heidelberg, Heidelberg, Germany

4 Institute of Reconstructive Neurobiology, Life and Brain Center, University of Bonn, Bonn, Germany

5 Division of Neuroradiology, Department of Radiology, University of Bonn, Bonn, Germany

6 Department of Neurology, University of Zurich, Zurich, Switzerland

7 Department of Neuropathology, Heinrich Heine University Düsseldorf, Düsseldorf, Germany

8 Department of Neuropathology, University of Bonn, Bonn, Germany

9 Department of Neurosurgery, University of Bonn, Bonn, Germany
10 Division of Molecular Genetics, German Cancer Research Center (DKFZ) Heidelberg, Heidelberg, Germany

11 Clinical Cooperation Unit Neuropathology, German Cancer Research Center (DKFZ) Heidelberg, Heidelberg, Germany

12 Department of Neuropathology, University of Heidelberg, Heidelberg, Germany

13 Department of Pediatric Oncology, Hematology and Immunology, University of Heidelberg, Heidelberg, Germany

14 German Cancer Consortium (DKTK), German Cancer Research Center (DKFZ) Heidelberg, Partner Site Essen/ Düsseldorf, Düsseldorf, Germany

15 Dr. Senckenberg Institute of Neurooncology, University of Frankfurt, Frankfurt, Germany

16 Department of Neurosurgery, University of Münster, Münster, Germany

17 Department of Neurology, Klinikum Ludwigsburg, Ludwigsburg, Germany 
Table 4 Univariate and multivariate Cox regression analysis regarding overall survival and single genetic factors

\begin{tabular}{|c|c|c|c|c|}
\hline & \multicolumn{2}{|c|}{ Median survival in months } & \multirow{2}{*}{$\begin{array}{l}\text { Risk ratio (lower-upper } 95 \% \mathrm{CI} \text { ) } \\
\text { Condition } 1 \text { vs. condition } 2\end{array}$} & \multirow[t]{2}{*}{$p$} \\
\hline & Condition 1 & Condition 2 & & \\
\hline \multicolumn{5}{|l|}{ Univariate analysis } \\
\hline$M G M T$ promoter status & $\begin{array}{l}\text { Methylated } \\
>37.1\end{array}$ & $\begin{array}{l}\text { Unmethylated } \\
8.9\end{array}$ & $0.09(0.02-0.44)$ & 0.004 \\
\hline IDH/CIMP status & $\begin{array}{l}\text { Mutant/positive } \\
36.3\end{array}$ & $\begin{array}{l}\text { Wildtype/negative } \\
14.1\end{array}$ & $0.31(0.08-1.23)$ & 0.08 \\
\hline $1 p / 19 q$ status & $\begin{array}{l}\text { Codeleted } \\
>16.5\end{array}$ & $\begin{array}{l}\text { Not codeleted } \\
34.8\end{array}$ & $0.44(0.05-3.6)$ & 0.44 \\
\hline Chromosome 7 gain & $\begin{array}{l}\text { Yes } \\
8.98\end{array}$ & $\begin{array}{l}\text { No } \\
>36.3\end{array}$ & $6.32(1.78-22.5)$ & 0.004 \\
\hline EGFR amplification & $\begin{array}{l}\text { Yes } \\
7.9\end{array}$ & $\begin{array}{l}\text { No } \\
36.3\end{array}$ & $10.1(2.0-51.1)$ & 0.005 \\
\hline 9 p21 deletion & $\begin{array}{l}\text { Yes } \\
37.1\end{array}$ & $\begin{array}{l}\text { No } \\
34.6\end{array}$ & $1.15(0.33-4.0)$ & 0.82 \\
\hline Chromosome 10 deletion & $\begin{array}{l}\text { Yes } \\
12.2\end{array}$ & $\begin{array}{l}\text { No } \\
36.3\end{array}$ & $2.6(0.77-8.73)$ & 0.12 \\
\hline$C D K 4$ amplification & $\begin{array}{l}\text { Yes } \\
36.2\end{array}$ & $\begin{array}{l}\text { No } \\
34.6\end{array}$ & $0.52(0.1-2.6)$ & 0.42 \\
\hline$M D M 2$ or $M D M 4$ amplification & $\begin{array}{l}\text { Yes } \\
14.1\end{array}$ & $\begin{array}{l}\text { No } \\
34.6\end{array}$ & $1.06(0.28-4.1)$ & 0.93 \\
\hline Multivariate analysis & & & & \\
\hline$M G M T$ promoter status & Methylated & Unmethylated & $0.16(0.03-0.95)$ & 0.04 \\
\hline IDH/CIMP status & Mutant/positive & Wildtype/negative & $1.27(0.14-11.6)$ & 0.83 \\
\hline Chromosome 7 gain & Yes & No & $4.2(0.49-36.3)$ & 0.19 \\
\hline EGFR amplification & Yes & No & $3.0(0.56-16.3)$ & 0.20 \\
\hline
\end{tabular}

Significant $p$ values lower than 0.05 are in bold

Table 5 Univariate Cox regression analysis regarding the prognostic value MRI features for overall survival

\begin{tabular}{llll}
\hline MRI appearance & HR & $95 \%$ CI & $p$ \\
\hline Infratentorial involvement, yes vs. no & 0.4 & $0.05-3.1$ & 0.38 \\
Involvement of 6 or more lobes, yes vs. no & 1.08 & $0.33-3.5$ & 0.9 \\
Bilateral involvement, yes vs. no & 0.67 & $0.08-5.4$ & 0.71 \\
Contrast enhancing lesions ${ }^{\text {a }}$, yes vs. no & 1.39 & $0.37-5.3$ & 0.63
\end{tabular}

$H R$ hazard ratio, $C I$ confidence interval

${ }^{\text {a }}$ On the first MRI fulfilling the criteria of GC 